\title{
Supramolecular Dynamics of Mucus
}

\author{
Pedro Verdugo \\ Friday Harbor Laboratories, University of Washington, Friday Harbor, Washington 98250 \\ Correspondence: verdugo@u.washington.edu
}

Our purpose here is not to address specific issues of mucus pathology, but to illustrate how polymer networks theory and its remarkable predictive power can be applied to study the supramolecular dynamics of mucus. Avoiding unnecessary mathematical formalization, in the light of available theory, we focus on the rather slow progress and the still large number of missing gaps in the complex topology and supramolecular dynamics of airway mucus. We start with the limited information on the polymer physics of respiratory mucins to then converge on the supramolecular organization and resulting physical properties of the mucus gel. In each section, we briefly discuss progress on the subject, the uncertainties associated with the established knowledge, and the many riddles that still remain.

\section{A ROAD MAP}

$M$ ucus has a complex set of functions in the airway, including its central role in mucociliary clearance. Defective mucus flow rests at the base of some of the most critical pulmonary pathology, including chronic obstructive pulmonary disease (COPD), cystic fibrosis (CF), and asthma. Research on the intricate mechanisms that regulate mucus rheology, particularly in CF, has been largely focused on mucins and on the defective function of the cystic fibrosis transmembrane conductance regulator (CFTR). The biochemistry and molecular biology of mucins (Perez-Villar and Hill 1999; Chen et al. 2001; Thornton et al. 2008) and the biophysics of the CFTR (Riordan 2005; Sheppard and Welsh 2006) have all received a great deal of attention during this last decade. Even so, the pathophysiology of defective mucus remains largely phenomenological, and an understanding of the mechanisms that make airway mucus transportable is still limited. Mucus is a polymer gel, and this question rests by and large in the domains of the physics and physical chemistry of polymer gels. The matrix of polymer gels forms a supramolecular network with emerging properties that cannot be understood only on the basis of the properties of the molecules that make it. When polymers are constrained to lie close together by physical or chemical bonds, their behavior departs from those of free polymers. A new set of features emerges that is largely determined by polymer-polymer and polymer-solvent interactions, as well as the topological features of the gel's matrix, including the nature and density of interconnections (low/high energy bonds and physical tangles) and the conformational state and mechanical properties of the polymers that make it. Nonetheless, most of the emphasis on mucus research remains focused on the biochemistry and molecular biology of mucins, the giant polymers found in the mucus gel matrix.

Editors: John R. Riordan, Richard C. Boucher, and Paul M. Quinton

Additional Perspectives on Cystic Fibrosis available at www.perspectivesinmedicine.org

Copyright (C) 2012 Cold Spring Harbor Laboratory Press; all rights reserved; doi: 10.1101/cshperspect.a009597

Cite this article as Cold Spring Harb Perspect Med 2012;2:a009597 
P. Verdugo

One of the oldest controversies in mucus research questions the nature of interconnections among mucin chains: whether the mucus is a chemical gel with a matrix containing branched polymers interconnected by covalent $\mathrm{S}: \mathrm{S}$ bonds, or a physical gel in which linear polymers are held together by tangles and lowenergy electrostatic and hydrophobic bonds (Lee et al. 1977; Clamp et al. 1978; Verdugo et al. 1983). Because the rheological properties of chemical gels depend on the degree of covalent cross-links among polymer chains (Flory 1953), defective control of S:S bonding was thought to be the source of mucus pathology (Clamp et al. 1978). Conversely, the rheological properties of physical gels depend on the degree of swelling of the gel's matrix, that is, the number of tangles per unit of gel volume (Edwards and Grant 1973a). Mucus pathology in this case is likely to result from defective swelling (Verdugo 1984, 1990, 1991, 1998; Chen et al. 2010). The demonstration that mucus remains condensed while stored in the granule to then undergo massive swelling upon exocytosis (Verdugo 1984, 1991) validated the argument that swelling is a necessary and sufficient condition for mucus to reach its normal flow properties. It also rendered this controversy largely moot because, chemically or physically interconnected, the mucus gel must swell to become transportable because in either case it emerges from a condensed conformation. Mucus pathology in either case is likely to result primarily from defective swelling - with one caveat: if the mucus matrix is covalently bonded, swelling will reach a limit and eventually expand to a maximum volume determined by the degree of interchain (intermolecular) bonding (Flory 1953); the more the bonding, the less it can swell and the harder it should be to transport. However, there no limit to swelling if the matrix is tangled. The mucus matrix will freely relax to reach an equilibrium swelling that is controlled only by the amount of available water and particularly the composition of its solvent (e.g., the ASL). Because the rheological properties of tangled-matrix gels depend on tangle density (Edwards and Grant 1973a) and interchain tangles decrease exponentially as mucus volume grows (de Gennes and Leger 1982; Doi and Edwards 1984), small changes in swelling equilibrium result in large changes in mucus rheology, and deficient swelling could be a significant source of mucus pathology (Verdugo 1984, 1990, 1991). Defective mucus swelling became a central question in mucus pathology. Airway surface dehydration had been postulated to be the culprit (Matsui et al. 1998; Boucher 2007). However, regardless of the water supply, unless $\mathrm{Ca}$ is dislodged from the mucus matrix, mucus hydration will be drastically limited (Verdugo et al. 1987b; Aitken and Verdugo 1989; Verdugo 1998; Chen et al. 2010). The required physiological chelator responsible for withdrawing $\mathrm{Ca}$ from the mucus matrix turns out to be $\mathrm{HCO}_{3}$, providing a powerful basis to understand $\mathrm{CF}$ mucus pathology (Garcia et al. 2009; Chen et al. 2010; Muchekehu and Quinton 2010; Quinton 2010).

Direct evidence from EM, DLS, and AFM portray mucins as linear chains (Sheehan et al. 1984; Marianne et al. 1987; Gupta et al. 1990; Matthews et al. 2002; Round et al. 2002; Bansil et al. 2005; Hong et al. 2005). However, biochemical evidence indicates that mucins in gastrointestinal mucus might be S:S-bonded (Ambort et al. 2011). These different matrix topologies may reveal two corresponding strategies of mucus function, which are particularly adapted to different local conditions of the guts and airways. In the gastrointestinal tract, a dense layer of protective mucus is required to defend the mucosa from being digested, and because mucus is exposed to large volumes of solvent, it seems fitting to limit mucus swelling to preserve a tight protective molecular mesh. In the airway, mucociliary flow requires delicate dynamic regulation of mucus rheology. This is probably achieved by strict control of mucus swelling equilibrium by managing water movement across the mucosa and delicate control of $\mathrm{Na}, \mathrm{pH}$, and particularly of $\mathrm{Ca}$ and $\mathrm{HCO}_{3}$ concentrations in the ASL. An additional level of complexity is that in chronic airway pathology, the polymer tangled topology of airway mucus might become covalently cross-linked (Thornton et al. 1991; Sheehan et al. 1999). In this case, the normal mechanisms that control 
mucus hydration would become ineffective in restoring normal mucus flow properties.

We first introduce a definition of polymer gels and give a few words regarding emergence in polymer networks. Gels are formed by a three-dimensional (3D) polymer matrix embedded in a solvent, which is water in the case of biological hydrogels. Solvent prevents the collapse of the network, and the network entraps the solvent, creating microenvironments that are in thermodynamic equilibrium with the surrounding media. "Gels" are a unique form of hierarchically structured supramolecular organization in which the polymers that form the gel matrix form a 3D network interconnected by chemical or physical cross-links that keep these chains in a statistically stable close neighborhood (Tanaka 1981). The chemical and physical characteristics of the individual polymer chains (including polyelectrolyte properties, hydrophobicity, size, linear or branched structure, etc.) and the nature of their interconnections determine the topology, chemical reactivity, and bulk physical properties of gel networks and how gels interact with solvents, smaller solutes, and microorganisms. Covalently cross-linked chemical gels have a defined limit swelling volume and cannot anneal. That is, polymers are tightly bound to each other and cannot migrate out of their network to interpenetrate neighboring gels and anneal, forming larger gels. Conversely, in physical gels, polymers can axially slide past each other and swell indefinitely. Axial mobility is an important feature of tangled networks - namely, it allows polymers from neighboring gels to interpenetrate and anneal to form larger gels (de Gennes and Leger 1982). In response to subtle physical or chemical shifts of their environment both cross-linked and entangled gels can undergo abrupt reversible phase transitions from a flexible and highly permeable swollen phase to a dense collapsed phase, and vice versa (Tanaka et al. 1980). As pointed out below, this mechanism allows volume-occupying mucus to collapse inside the granule as a densely compacted network.

"Emergence" refers to new properties that emerge from the collective behavior of multiple interacting components. For instance, only hydrogen and oxygen and no other elements can make water, and only a few features of these gases are required for water synthesis. However, once they combine to make water, a unique set of new properties emerges. In fact, the emergent properties of water have little to do with the two gases that make it, and little can be predicted about the emergent properties of water by speculating on the properties of these gases. Complex multiscale systems like polymer gels are characterized by a hierarchy of molecular order in which the impact of individual molecules in the bulk physical properties of the system is strictly conditioned by their mutual association with the supramolecular gel matrix. Just a few of a broad range of properties of the polymer components are important for gel assembly and bulk properties. Here again, the emergent properties of gels are not found in their component polymer chains, nor can they be unequivocally predicted from the chemical/physical properties of their individual polymer components.

Important advances in mucin biochemistry and molecular biology portray a remarkable complexity of mucin expression. There is a broad range of genetic variations that is producing an ever-growing molecular taxonomy of mucins, making it extremely intricate and arbitrary to specify structure-function assignments of these polymers to predict the complex features of a mucus gel explicitly. These studies make up a body of excellent science, but only a fraction of it can serve to reliably-and even then to only partially-forecast the properties of respiratory mucus. Most of the relevant physical information pertaining to macromolecular dynamics required to predict the role of mucin polymers in determining the bulk properties of mucus is still missing.

\section{POLYMER PHYSICS OF AIRWAY MUCINS}

\section{Conformation of Mucin Chains}

The molecular structure of mucins is reviewed in detail in Ambort et al. (2012b). Herewith we focus only on those features required to explain 
P. Verdugo

the assembly of mucus networks. MUC5AC and MUC5B, the main structural polymers in the matrix of airway mucus, are among the largest biopolymers known. They have a random-coil conformation in solution and are broadly polydispersed with a molecular mass ranging from $2 \times 10^{6}$ to $1 \times 10^{9} \mathrm{Da}$ and a chain (contour) length varying between $500 \mathrm{~nm}$ and several micrometers (Matthews et al. 2002; Round et al. 2002; Thornton et al. 2008; Kesimer et al. 2010). They consist of a core apoprotein in which polyanionic glycosylated blocks containing sialic and sulfate ester terminals alternate periodically with hydrophobic "naked" cysteine-rich blocks. This pattern of alternating negatively charged hydrophilic blocks with globular hydrophobic blocks resembles the typical architecture of synthetic amphiphilic brush multiblock copolymers (Bates and Fredrickson 1990; Dobrynin and Rubinstein 2005). It gives mucins a broad potential for folding and forming ordered collapsed structures. Among the most critical characteristics of mucins for their role in respiratory mucus is their linear, unbranched conformation. Early work using dynamic laser scattering (DLS) revealed that mucin chains in cervical and respiratory mucus can diffuse along their axis (Lee et al. 1977; Verdugo et al. 1983). In chemically cross-linked gels, polymers can show only lateral local motion associated to the chain sections between crosslinks (Doi and Edwards 1984). However, in entangled gels, polymers can diffuse both locally and axially, the latter resembling the motion of a snake inside a randomly convoluted tube. These random movements are reported by two corresponding DSL sets of relaxation times-namely, short relaxation times reflecting limited local lateral polymer mobilities and long relaxation times associated with translational diffusion along the polymer axis that de Gennes and Leger (1982) called "reptation." DSL revealed that both sets of random diffusion mobilities are present in cow estrous cervical mucus (Lee et al. 1977) and in human bronchial mucus (Verdugo et al. 1983). Because reptation cannot take place in networks held together by covalent cross-links or made out of branched polymers (Edwards and Grant 1973b; de Gennes and
Leger 1982; Doi and Edwards 1984), these results provided the first objective indication that the polymer matrix of respiratory and cervical mucus is made of linear mucins that most likely are not chemically cross-linked but are tangled, forming a loosely woven network. Early EM images (Sheehan et al. 1984; Marianne et al. 1987) and more recent AFM imaging methods confirmed that respiratory MUC5B and MUC5AC show a characteristic linear conformation with contour lengths that can reach several micrometers (Gupta et al. 1990; Round et al. 2002; Bansil et al. 2005; Hong et al. 2005). Recent work using DLS and viscometry indicates that purified pig gastric mucins resemble an S:S-linked linear collection of dumbbell units forming daisy chain-like linear polymers (Yakubov et al. 2007). Similar results were reported by Bansil et al. (2005). DLS results in human mucins (Gupta et al. 1990) agree with the model that the molecular conformation of mucins consist of subunits connected by covalent (disulfide) bonds forming linear chains.

Mucins' linear conformation and large size are critical features for the airway mucus matrix. Swelling expands tangled networks requiring polymers to slide past each other, making linear conformation a paramount condition for axial diffusion and expansion. Regarding polymer size, the stability of tangled networks depends on the second power of the contour length of the polymers that form it (de Gennes and Leger 1982). This is because to move inside the network, polymers must diffuse axially, to "reptate" their way through multiple tangles, like a randomly wriggling snake. Because random walk times are proportional to the second power of the distance traveled (in this case, the contour length of the snake), small changes of mucin length result in drastic changes in mucus swelling kinetics. Upon exocytosis, mucus undergoes massive quick swelling, and mucin chain length can be a major hindrance to network relaxation (expansion). Thus, small changes of mucin length result in dramatic changes in swelling rate and swelling equilibrium. Conversely, mucin shortening by cleavage of S:S bonds of the apomucin backbone can readily disperse 
the mucus matrix. Enzymes immobilized inside the condensed mucus granule could be activated following exocytosis and potentially play a role in peptide cleavage in mucus conditioning (J Sheehan, unpubl.). Unfortunately, the actual size of mucins in their native state remains uncertain. Biochemical separation procedures result in broad polydispersity due to extensive cleavage yielding molecular dimensions that are far from their original size and conformation (Sheehan and Thornton 2000; Round et al. 2002). Recent developments of methods to isolate secretory granules (Marszalek et al. 1997) allow working with uncontaminated material released from isolated electroporated granules. Mucin polymers released from isolated goblet cell granules spontaneously dispersed onto $\mathrm{Ca}$ free, $140 \mathrm{~mm} \mathrm{Na}, \mathrm{pH} 6$ medium. They show a narrow $0.1-0.3$ polydispersity with mean square radius of gyration $R_{\mathrm{g}} \approx 230 \mathrm{~nm}$ (EY Chen and WC Chin, pers. comm.). These large dimensions agree with DSL results in salivary mucins (Kesimer et al. 2010) and with the multimicrometer contour length observed by atomic force microscopy in ocular and airway MUC5AC (Matthews et al. 2002; Round et al. 2007). In summary, despite their critical roles in mucus bulk properties, the contour length and solvent composition-dependent conformation of mucin chains in respiratory mucus remain uncertain.

\section{Polyelectrolyte and Hydrophobic Features of Mucins}

Sialic and sulfated sugar residues make mucins strongly polyanionic with an isoelectric $\mathrm{pH}$ point of $\sim 2.5$ (Bansil et al. 1995; Thornton et al. 2008). This feature has two important implications. First, it means that swelling of mucus must be driven by fixed charges and governed by a Donnan process (Katchalsky et al. 1951), an issue validated (Tam and Verdugo 1981) and addressed in detail in the next section on polymer physics of the mucus gel. Second, the elastic properties and equilibrium conformation of polyelectrolytes depend very strongly on the dielectric properties, counterion composition, and $\mathrm{pH}$ of the solvent. Depending on solvent composition, polyelectrolytes can be flexible or rigid and brittle. These features can directly affect the rheological properties of the gels they form (Doi and Edwards 1984). Theoretically, the polyanionic charge of mucins makes the conformation, hydrodynamic diameter, and, especially, the elastic properties of these polymers highly sensitive to $\mathrm{pH}$ and to cations, particularly $\mathrm{Ca}$ and $\mathrm{Na}$ present in the ASL. Atomic force microscopy (AFM) reveals that conformation and folding of gastric and ocular mucin chains are critically dependent on pH (Bansil et al. 1995; Round et al. 2004). In summary, several lines of evidence confirm that airway mucins show a linear conformation and large molecular dimensions. However, the effects of $\mathrm{Ca}, \mathrm{Na}$, and $\mathrm{pH}$ on polymer elasticity, folding, and conformation of airway mucins have not been conducted. It is likely that mucins will undergo globule/coil transition upon exposure to solvent compositions that mimic those that induce macroscopic condensation of the mucus gel, namely, a low $\mathrm{Na} / \mathrm{Ca}$ ratio and $\mathrm{pH}$ (Verdugo et al. 1992; Verdugo 1994). Direct evaluation of the effect of solvent composition on airway mucin conformation is an important still pending task, because change of polymer elasticity can directly affect the viscoelastic properties of mucus.

Direct measurements of mucin zeta $(\zeta)$ potential to assess changes in polyanionic charge density have not been performed in undegraded respiratory mucins at different $\mathrm{Na} / \mathrm{Ca}$ ratios and $\mathrm{pH}$. This information is crucial because variations of charge density of mucins could strongly affect the mucin electrostatic interactions and bulk properties of mucus, as well as bacterial binding to mucus (van Loosdrecht et al. 1990; Rayner and Wilson 1997).

Equally unexplored is the effect of small anions on the conformational dynamics of mucin polymers. Most of the few advances have been focused on effects of salt cations, whereas the anions of the supporting electrolyte have been largely ignored. Direct or indirect effects of anions on mucus, particularly by $\mathrm{HCO}_{3}$, have only recently begun to be systematically investigated, revealing the crucial role of this anion on mucus swelling equilibrium (Garcia et al. 
P. Verdugo

2009; Chen et al. 2010; Muchekehu and Quinton 2010; Quinton 2010).

Finally, the role of hydrophobic interactions in mucus-bacteria adhesion is well established (Rayner and Wilson 1997). Hydrophobic interactions play critical roles on gastric mucin conformation, folding, and interchain binding (Bansil et al. 1995). However, similar studies in respiratory mucins have not been conducted.

\section{Mucin Polymer Folding}

Hydrated mucins are volume-occupying polymers, well suited to form the matrix of mucus. However, while in storage the mucus gel remains in a condensed phase (Verdugo et al. 1992, 1994) with mucin chains highly compacted and constrained within a drastically limited intragranular volume. This state is hardly compatible with a simple collapsed random-coil conformation. Mucins, however, have the characteristic features of triblock copolymers with comb glycosylated hydrophilic rigid blocks flanked by flexible globular hydrophobic blocks (Di Cola et al. 2008; Waigh and Papagiannopoulos 2010). A characteristic feature of block copolymers is that they can fold to form ordered compact metastable liquid crystalline phases (Halperin 1991). Nematic crystalline ordered domains are, indeed, present in the mucus of the banana slug Ariolimax columbianus (Viney et al. 1993a,b). Mucus released from slug granules can swell at virtually explosive rates, going from 4 to $300 \mu \mathrm{m}$ diameter in $\sim 20 \mathrm{~ms}$, with a corresponding volume increase close to $4.2 \times$ $10^{4}$ times their condensed size (Verdugo et al. 1992). At this rate of expansion, the internal frictional dissipations of polymers sliding with respect to one another would be enormous. Unfolding of ordered mucin blocks present between tangles can explain how the mucin matrix of these granules undergoes explosive swelling expansion with little reptational dissipation that otherwise would be required if mucins were to form a random network (Fig. 1). Thus, nematic ordered phases may account for both high-density folded packing and for reducing internal shear dissipation upon swelling via an unfolding of ordered mucin blocks. Important features of block copolymers predicted by theory are starting to be verified in synthetic block copolymer networks (Bates and Frederickson 1990) and are critical to understand the complex polymer dynamics of mucin gels. Verification with regard to respiratory mucus remains urgently important unfinished work.

In summary, the biochemistry and molecular biology of respiratory mucins have produced a rich bounty of data. Unfortunately, however, most of this information otherwise rich in implications for many aspects of mucins functions is of limited significance to the network formation and supramolecular dynamics that are essential to predict mucus rheology in the airways. We are still missing fundamental pieces of the puzzle to build a precise multidimensional phase diagram of mucin-size dynamics and changes of conformation resulting from exposure to different $\mathrm{Na} / \mathrm{Ca}$ ratios, $\mathrm{pH}, \mathrm{HCO}_{3}$, temperature, and the presence of amphiphilic and organic polycations normally found in the ASL. This information is critical to predict mucin interactions within the mucus matrix.

\section{PHYSICS OF THE MUCUS GEL}

\section{Diffusive Mobilities of Mucins in the Mucus Matrix}

We now turn our attention from the physics of mucin chains to the physics of the mucus matrix. As pointed out above, results from DLS studies in cow cervical mucus (Lee et al. 1977) and in human bronchial mucus sampled by bronchoscopy (Verdugo et al. 1983) showed the presence of reptational axial diffusion. These results ruled out the idea that these mucins are held together by interchain covalent bonds (de Gennes and Leger 1982) and provide strong support for the hypothesis that mucus is a physical gel held together by tangles and lowenergy interactions. Because the rheological properties of gels containing a tangled matrix depend on tangle density, which decreases with the square of the gel volume (Edwards and Grant 1973b; de Gennes and Leger 1982), these observations implied that swelling should be the primary influence on mucus rheology, with a 

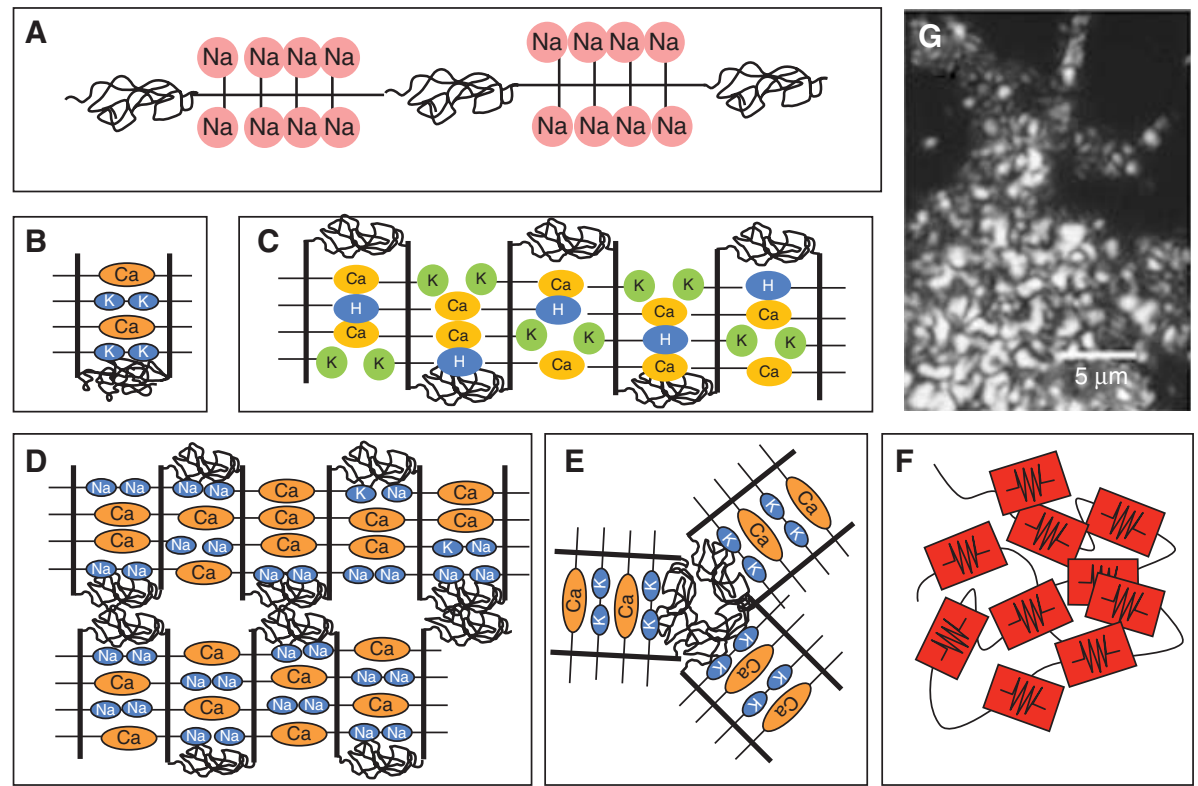

Figure 1. Block copolymer model for airway mucus. The typical periodic hydrophilic/hydrophobic domains and lineal conformation give airway mucins the characteristic profile of block copolymers. The elementary length structure in this model is a binary amphiphilic triblock copolymer consisting of alternating hydrophilic glycosylated polyanionic blocks_-resembling densely brush-grafted blocks-flanked by globular peptide hydrophobic blocks (Waigh and Papagiannopoulos 2010). (A) In extended conformation-outside the granuleblocks are interconnected forming large linear chains with contour lengths reaching up to several micrometers. (B) While inside the granule, chains are condensed with polyanionic glycosylated blocks fully shielded by counterions and folded forming loops stabilized by $\mathrm{H}$ and Ca links. $(C)$ These $\mathrm{U}$-shaped blocks are interconnected, forming long densely collapsed lattices. $(E)$ Lattices can associate laterally via hydrophobic bonds and assemble into large supramolecular nematic liquid crystalline ordered arrays as shown in $D$ (planar view) that in side view may hold triplet or even quadruple interlinked collapsed chains. (F) Supramolecular arrays, like those illustrated in $E$, are interconnected, forming a tangled random network. In condensed phase, water inside the matrix is constrained to within the Debye field of the polymer charged sites, with a dielectric permittivity that keeps counterions bound. Upon formation of the secretory pore, release of protons and entry of water into the gels matrix result in a drastic change of dielectric property, changing long-range solvent-mediated dynamic interactions among blocks, triggering high-cooperativity counterion dissociation from binding sites. Donnan swelling - resulting from $\mathrm{Na} / \mathrm{Ca}$ exchange-results in splitting Ca cross-links and repulsive polyanionic interactions that quickly unfold the mucin arrays (represented by boxes). The ordered topology of the network (expansion of the boxes) allows fast isotropic low-dissipative spreading out of the mucus chains without forming knots and loops that would otherwise be expected in a randomly tangled matrix expanding only on the basis of polymer reptation. (Modified from a napkin sketch drawing during our last sushi brainstorming session with my late friend Toyo Tanaka in Boston, early May 2000.) (G) An actual polarized microscopy micrograph of nematic liquid crystalline formation in mucus (Viney et al. 1993a).

quadratic dependence further implying that slight swelling deficits should result in thick, highly viscous non-transportable mucus (Verdugo 1990, 1991). They further suggested that mucus must start from an unswollen state, opening the chase for the physical-chemical control of mucus swelling.

\section{Donnan Control of Mucus Swelling}

In polymer gels, interchain bonds and tangles constrain the mobility of polymers and keep them inside the gel matrix. Swelling of gels is driven by forces generated by osmotic pressure; that is, if the chains forming the gel's matrix 
P. Verdugo

generate higher osmotic pressure than solutes in the bulk solvent outside the gel, the gel swells. In gels made of neutral polymers, the swelling rate is slow because, in this case, osmotic pressure results from limited hindered diffusion of the large polymers that form the gel's matrix. However, in gels made of polyionic polymers, their ionized charged sites are fixed and cannot move out of the matrix, because they are part of the polymers. As a result, electroneutrality holds small mobile counterions inside the matrix. A Donnan equilibrium is established (Katchalsky et al. 1951). In this case, higher concentrations of mobile ions inside the gel than in the bulk solvent outside the gel generate almost all of the osmotic pressure that drives quick swelling. Swelling equilibrium is reached when the elastic properties of the matrix balance the expanding forces generated by osmotic pressure. Katchalsky's findings (Katchalsky et al. 1951) that in ion exchange gels, swelling is driven by a Donnan potential, led the way to verify that mucus hydration is governed by a Donnan equilibrium process (Tam and Verdugo 1981). These observations gave the first objective physical-chemical foundation for understanding the role of solvent composition in the regulation of mucus swelling (Verdugo 1984, 1990, 1991). The significance of these observations became apparent following our discovery that the mucus gel is stored in a highly compacted form undergoing swelling following its release from the cell.

\section{Mucus Polymer Gel Phase Transition}

The experimental demonstration that mucus, like all polymer gels, can undergo reversible phase transition from a condensed to a solvated phase (Verdugo et al. 1992; Verdugo 1994) provided a strong indication that Duŝek-Tanaka's theory (Duŝek and Patterson 1968; Tanaka et al. 1980; Tanaka 1981) can, indeed, be applied to understand the mechanisms for mucus storage in and release from secretory granules (Verdugo et al. 1992; Verdugo 1994). Phase transition is a characteristic property of all polymer gels. Duŝek-Tanaka's theory establishes that gels can exist in either a condensed or a solvated phase. Just as transitions of liquids to solids or to gas are reversible and occur at precise critical temperatures and pressures, a polymer gel phase transition (PGPT) from a condensed to a solvated phase occurs within a narrow range of critical conditions, shows high cooperativity, and is fully reversible (Tanaka et al. 1980; Tana$\mathrm{ka}$ 1981). The polymer matrices of most secretory granules including mucins, chromogranins, heparin, SP-1, and the like have the typical features of polyionic polymer gels. During storage in secretory granules, the matrix remains in condensed phase, in an intragranular environment of high Ca and low pH (Izutsu et al. 1985; Verdugo et al. 1987a; Villalon et al. 1988). Condensation is not a simple process of dehydration. The matrix of isolated goblet and mast cell granules remains condensed in water for as long as the ionic composition of the medium mimics intragranular environment, that is, low $<500 \mu \mathrm{M} \mathrm{Na}$, high $>10 \mathrm{~mm} \mathrm{Ca}$, and $<6 \mathrm{pH}$. However, exposure to solutions containing 140 $\mathrm{mm} \mathrm{Na}, 2 \mathrm{~mm} \mathrm{Ca}$ at $\mathrm{pH}$ 7, which may mimic ASL, triggers PGPT and quick matrix swelling (Fernandez et al. 1991; Verdugo et al. 1992; Verdugo 1994). Moreover, swollen mucus can be readily recondensed by exposure to solutions that mimic the intragranular environment (Verdugo 1991, 1994; Verdugo et al. 1992). In the secretory cell, following the formation of a secretory pore, protons must quickly escape to the extracellular medium, and $\mathrm{Ca}$ inside the network must be rapidly exchanged by $\mathrm{Na}$ (Forstner and Forstner 1975; Izutsu et al. 1985; Villalon et al. 1988). Because negative charges of the mucins are fixed (cannot move out of the matrix), exchange of divalent Ca by monovalent Na causes the gel to swell. Donnan swelling in this case results from doubling the number of counterions, as required by electroneutrality (two $\mathrm{Na}$ atoms for each $\mathrm{Ca}$ ). An increased number of ions inside the gel results in increased osmotic pressure and swelling. With one caveat, ASL Ca should remain low (Verdugo et al. 1987b; Verdugo 1998). The missing chelating agent that buffers $\mathrm{Ca}$ in the ASL was found to be $\mathrm{HCO}_{3}$, providing a straightforward explanation for the mucus pathology in CF (Garcia et al. 2009; Chen et al. 2010; Muchekehu and Quinton 2010; Quinton 2010). 
In airway goblet cells, the matrix of exocytosed mucus undergoes quick swelling, forming microspheres that increase their diameter from $\sim 1 \mu \mathrm{m}$ to $\sim 4-5 \mu \mathrm{m}$ with $\sim 6.25$ folds or $625 \%$ volume expansion in 3-5 s (Verdugo 1984, 1998; Verdugo et al. 1987b). Fast swelling in a randomly tangled network should result in highly frictional dissipations and anisotropic expansion, resulting in tight knots and large loops. Instead isotropic mucus swelling strongly suggests the presence of ordered domains that can readily unfold in the mucus matrix. This feature was soon confirmed by the demonstration of nematic liquid crystalline order in mucus (Viney et al. 1993a,b), and set the basis for the model illustrated in Figure 1. Nematic anisotropies were observed by polarized light at visible wavelength and reveal that mucus ordered clusters are extremely large. Compacting of the matrix during storage inside the granule is likely to require a higher order. Based on EM images, Ambort et al. (2012a) have proposed a new model for mucin folding. However, inherent fixation artifacts weaken the significance of the proposed model, leaving mucin-folding topology still unresolved (Verdugo 2012). X-ray diffraction studies to verify ordered supramolecular mucin assembly in isolated mucus granules are unfortunately still missing.

A rich body of available theory-well verified in synthetic block copolymer networkscan be used to explain the characteristic ordered supramolecular arrays responsible for nematic features found in mucus (Halperin 1991). Ordered folding of mucins permits high-density packing and quick expansion of the network upon release from the cell. $\mathrm{Ca}$ is required to shield charges and cross-link the folded condensed phase of the mucus matrix. Upon release from the granule, $\mathrm{Na} / \mathrm{Ca}$ exchange thrusts Donnan-driven swelling and releases Ca crosslinks, unraveling the mucin network. Thus, an adequate supply of water (Matsui et al. 1998; Boucher 2007) and particularly the removal of Ca from the ASL are critical for normal mucus hydration (Verdugo et al. 1987b; Aitken and Verdugo 1989; Verdugo 1998; Garcia et al. 2009; Chen et al. 2010; Muchekehu and Quinton 2010; Quinton 2010).

\section{Mucus Swelling Kinetics during Product Release in Exocytosis}

Swelling is a necessary and sufficient condition for respiratory mucus to become transportable, and physical theories provide testable predictions for its regulation. However, experimental models to validate these ideas became available only after the introduction of primary tissue culturing methods of airway mucosa, allowing direct monitoring of ciliary activity and mucus exocytosis (Verdugo 1980, 1984). In agreement with Tanaka and Fillmore's (1979) theory of swelling of polymer gels, the volume expansion of exocytosed mucus follows a typical firstorder kinetics (Fig. 2). It has the distinctive features of a diffusion process in which the characteristic relaxation time of expansion is proportional to the second power of the linear dimension, in this case, the increase of the radius of the exocytosed swelling mucus matrix (Verdugo 1984, 1998; Verdugo et al. 1987b; Aitken and Verdugo 1989; Fernandez et al. 1991; Dodd et al. 1998; Espinosa et al. 2002; Chin et al. 2006; Chen et al. 2010). The slope of this line has the dimensions of centimeters squared per second $\left(\mathrm{cm}^{2} / \mathrm{s}\right)$ and corresponds to the diffusivity $(D)$ of the gel in the solvent, which in the case of airway mucus is the ASL.

Diffusivity $(D)$ is an extremely sensitive parameter for assessing mucus swelling resulting from changes of composition of the ASL like $\mathrm{pH}, \mathrm{Na} / \mathrm{Ca}$ ratio, organic polycations, anions, and the like (Verdugo et al. 1987b; Aitken and Verdugo 1989; Verdugo 1998; Espinosa et al. 2002; Chen et al. 2010). Measurements of swelling kinetics during exocytosis in primary cultures of airway rabbit goblet cells rendered the first indication that $\mathrm{pH}$ changes in the direction of the mucin isoelectric point can-as predicted by theory-decrease the mucus-swelling rate. Changes of $\mathrm{pH}$ from 7.5 to 7 , and 6.5 in a solvent containing $140 \mathrm{~mm} \mathrm{Na}$ and $1.5 \mathrm{~mm} \mathrm{Ca}$, results in changes of $D$ from $1.2 \times 10^{-7}$ to $4 \times 10^{-8}$ and $1 \times 10^{-8} \mathrm{~cm}^{2} / \mathrm{s}$, respectively, with a corresponding decrease of mucus swelling equilibrium that ultimately establishes the micro rheological load on cilia (Verdugo 1984). Collapsing the ASL-mucus gel Ca gradient-in this 


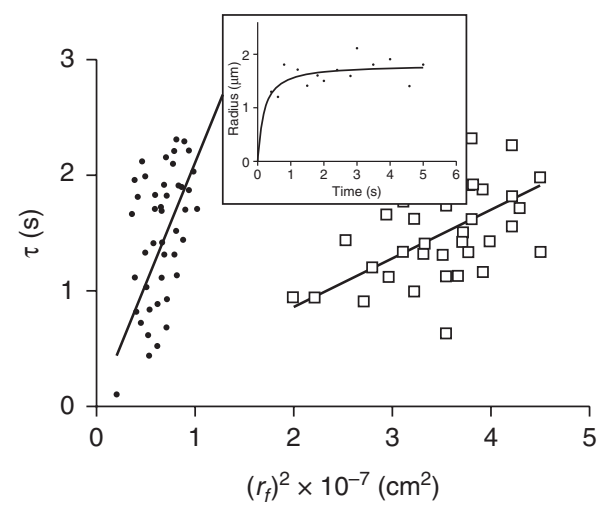

Figure 2. Illustrated here are data from studies published earlier (Verdugo 1998). Measurements from digitized video images show that the radial expansion of exocytosed goblet cell granules follows characteristic first-order kinetics, lending the process to be formalized in light of Tanaka's theory of swelling of polymer gels (Tanaka and Fillmore 1979). (Inset) A typical plot in which the continuous line is a nonlinear least-squares fitting of the data points to $r(t)=$ $r_{f}-\left(r_{f}-r_{i}\right) \times e^{-t / \tau}$, where $r_{i}$ and $r_{f}$ are the initial and final radii of the granule and $\tau$ is the characteristic relaxation time of swelling, with $r_{i}$ assumed to be $0.25 \mu \mathrm{m}$. Data points were collected as soon as the mucus micro gels became visible in phase contrast microscopy images. Notice that the square of the final radius is a linear function of $\tau$. The slope of this line has the characteristic dimensions of diffusion $\left(\mathrm{cm}^{2} /\right.$ $\mathrm{s}^{-1}$ ), and, according to Tanaka's theory, it reflects the diffusion of the gel in the solvent. Mucus granules exocytosed by cultured goblet cells from biopsied nasal polyps of normal subjects and from CF patients swell when cell equilibrated in Hanks' solution containing $1 \mathrm{~mm} \mathrm{Ca}$ ( $\mathrm{pH}$ 7.2). $D$ in normal mucus (open squares) reached $3.1 \pm 1.2 \times 10^{-7} \mathrm{~cm}^{2} / \mathrm{s}$. CF mucus granules swelled at almost an order of magnitude slower than normal (closed circles), yielding a $D=$ $7.4 \pm 2 \times 10^{-8} \mathrm{~cm}^{2} / \mathrm{s}$ and also reaching a much smaller final swelling volume equilibrium. These differences become much more pronounced when cells were equilibrated in Hanks' solution containing $2.5 \mathrm{~mm}$ Ca. In this case, exocytosed mucus from cells of normal subjects reached $D=2.5 \pm 0.9 \times$ $10^{-7} \mathrm{~cm}^{2} / \mathrm{s}$, whereas in CF mucus, $D$ reached only $9.1 \pm 1.8 \times 10^{-9}$. $D$ values correspond to the average \pm SEM of 96 exocytosis-swelling events. These observations suggest that, in addition to the CF defective $\mathrm{Ca}-\mathrm{HCO}_{3}$ chelation, it is likely that mucin-Ca affinity might be increased, resulting in decreased $\mathrm{Na} / \mathrm{Ca}$ exchange in CF. Alternatively, hydrophobic bonding, still largely unexplored, might be increased in CF. case, by increasing $\mathrm{Ca}$ in the medium-must prevent removal of Ca cross-links among mucin polymers and inhibit mucus swelling. The experimental results showed a decrease of $D$ from $2.5 \times 10^{-7}$ to $2 \times 10^{-8} \mathrm{~cm}^{2} / \mathrm{s}$ when $\mathrm{Ca}$ in the bathing medium increased from 1 to $4 \mathrm{~mm}$ in $140 \mathrm{~mm} \mathrm{Na}$ at neutral pH (Verdugo et al. 1987b). Recent experimental results confirm that increased extracellular Ca can drastically decrease both the swelling kinetics and swelling equilibrium in cervical mucus (Espinosa et al. 2002).

Measurements of $D$ to investigate mucus pathology revealed that in goblet cells from CF patients, $\mathrm{Ca}$ inhibition of mucus swelling is about five times stronger than in goblet cells from normal individuals (Verdugo 1998). These observations provided the first objective demonstration that in human goblet cells, ASL $\mathrm{Ca}$ can inhibit mucus swelling, and that $\mathrm{Na}$ / Ca exchange in CF mucus might be defective. Considering that the payload of goblet cell granules includes large amounts of $\mathrm{Ca}$ that is released to the ASL during exocytosis (Izutsu et al. 1985; Villalon et al. 1988), chelation of ASL Ca is a critical requisite for normal mucus swelling (Verdugo et al. 1987b; Verdugo 1998). Recent observations show that $\mathrm{HCO}_{3}$ can function as the actual chelator of $\mathrm{Ca}$, suggesting that defective mucosal control of $\mathrm{HCO}_{3}$ might be responsible for altered mucus rheology in CF (Garcia et al. 2009; Chen et al. 2010; Muchekehu and Quinton 2010; Quinton 2010). These results are consistent with early reports indicating that $\mathrm{Ca}$ is increased in the mucus of $\mathrm{CF}$ patients (Potter et al. 1967). The effect of polyions and particularly counterions on swelling kinetics increases with the second power of their valence (Ohmine and Tanaka 1982). In this regard, micromolar concentrations of organic polycations like cathelicidin, normally released by the airway epithelium (Felgentreff et al. 2006), or spermine or spermidine-the condensing agents of DNA — released with nucleic acids from dead cells or bacteria, could in theory cross-link the mucus matrix and drastically inhibit mucus swelling particularly in infected airways, and their role still awaits to be investigated. 


\section{Hydrophobic Interactions in Mucus}

Electrostatic interactions are just one among several low-energy interactions that could cross-link the mucus matrix. Equally important but less explored is the potential contribution of hydrophobic interactions (Meyer et al. 2006). Hydrophobic interactions have been investigated in gastric mucus (Bansil and Turner 2006) but remain virtually unexplored in airway mucus. In polyelectrolyte systems in salt solutions-like the mucus gel-intermolecular interactions become independent of polymer charge and remain proportional to the presence of amphiphilic moieties over a large range of concentrations (Diamant and Andelman 1999). Strong interactions are known to take place in polymer-surfactant cosolutes even at very low concentrations (Kuhn et al. 1998). Thus, the amphiphilic nature of mucins presents the likely prospect that hydrophobic interactions are at work in respiratory mucus, between mucins, between mucins and lipids present in mucus (Widdicombe 1987), or between mucins and other amphiphilic molecules like surfactants released by Clara cells (Griese 1999) or exopolymer substances (EPSs) released by bacteria in infected airway (Rayner and Wilson 1997). These issues represent significant gaps still to be investigated in airway mucus.

\section{CLOSING REMARKS}

Much remains to explore to fully understand and predict the complex supramolecular dynamics of mucus. Significant effort has been invested in studying many features of mucins that, although highly significant to molecular biology or biochemistry, have so far served largely as the basis of speculation in attempting to explain what makes airway mucus a transportable gel. These studies portray a remarkable complexity of mucin expression with a broad range of genetic variations that is producing an ever-growing molecular taxonomy of mucins, making it extremely intricate and arbitrary to specify structure-function assignments of these polymers to explicitly explain the complex features of a mucus gel. We are still missing critical features specifically relevant to the role of mucins in mucus function. Mucins' $\zeta$ potential, the size, conformation, and elastic properties of native undegraded mucins, all remain to be investigated. Equally unexplored is the supramolecular organization of the mucus polymer network. In tangled networks, it is the mesh topology of interwoven mucins that determines the relaxation dynamics of the gel upon hydration. Transition from a condensed to a solvated phase requires programmed unfolding of large mucin chains. However, we know little regarding airway mucus supramolecular topology. The critical role of ions on mucus swelling has been shown, but much remains to be explored, and the significance of hydrophobic interactions in airway mucus remains greatly neglected.

\section{ACKNOWLEDGMENTS}

Over the last 30 plus years of NIH and later NSF funding, our research program gained strength from a remarkable crew of students and postdoctoral fellows who contributed with ideas, created a joyful collegial but critical laboratory atmosphere, and conducted the bulk of the work reported here. I am particularly grateful to Dr. Patrick Tam, Dr. Wiley Lee, Dr. Moira Aitken, Dr. Manuel Villalon, Dr. Monica Orellana, Dr. Ivan Quezada, Dr. Thien Nguyen, and Dr. Wei Chun Chin. Many of the ideas we explored in the past were born out of enlightening discussions with my colleagues Alex Silberberg, Sir Sam Edwards, Ralph Nossal, Ferenc Horkay, John Sheehan, Bill Davis, Adrian Parsegean, and Karel Duŝek, and particularly my dear late friends Carol Basbaum and Toyo Tanaka. I am thankful to them all, for they pointed the compass to guide new exploration and exciting discoveries. Last but not least, this paper was kindly edited by Paul Quinton to whom I am indebted for his patience and many excellent suggestions.

\section{REFERENCES \\ * Reference is also in this collection.}

Aitken ML, Verdugo P. 1989. Donnan mechanism of mucin release and conditioning in goblet cells: The role of polyions. J Exp Biol 53: 73-79. 
P. Verdugo

Ambort D, Van der Post S, Johansson MEV, Mackenzie J, Thomsson E, Krengel U, Hansson GC. 2011. Function of the CysD domain of the gel-forming MUC2 mucin. Biochem J 436: 461-470.

Ambort D, Johansson MEV, Gustafsson JK, Nilsson HE, Ermund A, Johansson BR, Koeck PJ, Hebert H, Hansson GC. 2012a. Calcium and pH-dependent packing and release of the gel-forming MUC2 mucin. Proc Natl Acad Sci 109: $5645-5650$.

* Ambort D, Johansson MEV, Gustafsson JK, Ermund A Hansson, GC. 2012b. Perspectives on mucus properties and formation-lessons from the biochemical world. Cold Spring Harb Perspect Med doi: 10:1101/cshperspect.a014159.

Bansil R, Turner BS. 2006. Mucin structure, aggregation, physiological functions and biomedical applications. Curr Opin Coll Interf Sci 11: 164-170.

Bansil R, Stanley HE, Lamont JT. 1995. Mucin biophysics. Ann Rev Physiol 57: 635-657.

Bansil R, Cao X, Bhaskar KR, LaMont JT. 2005. Dynamic light scattering study of gelation and aggregation of gastric mucin. SPIE 2982: 116-125.

Bates FS, Fredrickson GH. 1990. Block copolymer thermodynamics: Theory and experiment. Annu Rev Phys Chem 41: 525-557.

Boucher RC. 2007. Evidence for airway surface dehydration as the initiating event in $\mathrm{CF}$ airway disease. J Intern Med 261: $5-16$

Chen Y, Zhao YH, Di YP, Wu R. 2001. Characterization of human mucin 5B gene expression in airway epithelium and the genomic clone of the amino-terminal and 5'-flanking region. Am J Respir Cell Mol Biol 25: 542-553.

Chen EY, Yang N, Quinton PM, Chin WC. 2010. A new role for bicarbonate in mucus formation. Am J Physiol Lung Cell Mol Physiol 299: L542-L549.

Chin WC, Quesada I, Steed J, Verdugo P. 2006. Modeling polyanion-Ca crosslinking in secretory networks: Assessment of charge density and bond affinity in polyanionic secretory networks. Macromol Symp 227: 89-96.

Clamp JR, Allen A, Gibbons RA, Roberts GP. 1978. Chemical aspects of respiratory mucus. Br Med Bull 34: 39-41.

de Gennes PG, Leger L. 1982. Dynamics of entangled polymer-chains. Annu Rev Phys Chem 33: 49-61.

Diamant H, Andelman D. 1999. Onset of self-assembly in polymer surfactant systems. Europhys Lett 48: 170-176.

Di Cola E, Yakubov G, Waigh TA. 2008. Double-globular structure of porcine stomach mucin: A small-angle X-ray scattering study. Biomacromolecules 9: 3216-3222.

Dobrynin AV, Rubinstein M. 2005. Theory of polyelectrolytes in solutions and at surfaces. Prog Polym Sci 30: 1049-1118.

Dodd S, Place GA, Hall RL, Harding SE. 1998. Hydrodynamic properties of mucins secreted by primary cultures of Guinea-pig tracheal epithelial cells: Determination of diffusion coefficients by analytical ultracentrifugation and kinetic analysis of mucus gel hydration and dissolution. Eur Biophys J 28: 38-47.
Doi M, Edwards SF. 1984. The theory of polymer dynamics. Oxford University Press, Oxford.

Duŝek K, Patterson D. 1968. Transition in swollen polymer networks induced by intramolecular condensation. $J$ Polym Sci B Polym Phys 6: 1209-1216.

Edwards SF, Grant JWV. 1973a. The effect of entanglements on the viscosity of a polymer melt. J Phys A 6: 1171-1180.

Edwards SF, Grant JWV. 1973b. Effect of entanglements on diffusion in a polymer melt. J Phys A 6: 1169-1185.

Espinosa M, Noé G, Troncoso C, Ho SB, Villalón M. 2002. Acidic $\mathrm{pH}$ and increasing $\mathrm{Ca}^{2+}$ reduce the swelling of mucins in primary cultures of human cervical cells. Hum Reprod 17: 1964-1972.

Felgentreff K, Beisswenger C, Griese M, Gulder T, Bringmann G, Bals R. 2006. The antimicrobial peptide cathelicidin interacts with airway mucus. Peptides 27: 3100-3106.

Fernandez JM, Villalon M, Verdugo P. 1991. Reversible recondensation of mast cell secretory products. Biophys $J$ 59: $1022-1027$.

Flory P. 1953. Principles of polymer chemistry. Cornell University Press, Ithaca, NY.

Forstner JF, Forstner GG. 1975. Calcium binding to intestinal goblet cell mucin. Biochim Biophys Acta 386: $283-$ 292.

Garcia MA, Yang N, Quinton PM. 2009. Normal mouse intestinal mucus release requires cystic fibrosis transmembrane regulator-dependent bicarbonate secretion. J Clin Invest 119: 2613-2622.

Griese M. 1999. Pulmonary surfactant in health and diseases: State of the art. Eur Resp J 13: 1455-1476.

Gupta R, Jentoft N, Jamieson A M, Blackwell J. 1990. Structural analysis of purified human tracheobronchial mucins. Biopolymers 29: 347-355.

Halperin A. 1991. On the collapse of multiblock copolymers. Macromolecules 24: 1418-1419.

Hong Z, Chasan B, Bansil R, Turner BS, Bhaskar KR, Afdhal NH. 2005. Atomic force microscopy reveals aggregation of gastric mucin at low pH. Biomacromolecules 6: $3458-$ 3466.

Izutsu K, Johnson D, Schubert M, Wang E, Ramsey B, Tamarin A, Truelove E, Ensign W, Young M. 1985. Electron microprobe analysis of human labial gland secretory granules in cystic fibrosis. J Clin Invest 75: 1951-1956.

Katchalsky A, Lifson S, Eisenberg HJ. 1951. Equation of swelling for polyelectrolyte gels. J Polymer Sci 7: 571-574.

Kesimer M, Makhov AM, Griffith JD, Verdugo P, Sheehan JK. 2010. Unpacking a giant glycoprotein polymer from its storage granule: The mechanism of MUC5B mucin expansion after cellular release. Am J Physiol Lung Cell Mol Physiol 298: L15-L22.

Kuhn PS, Levin Y, Barbosa MC. 1998. Complex formation between polyelectrolytes and ionic surfactants. Chem Phys Lett 298: 51-56.

Lee WI, Verdugo P, Blandau RJ. 1977. The molecular structure of cervical mucus: A reevaluation by laser light scattering spectroscopy. Gynec Invest 8: 254-266.

Marianne T, Perini JM, Lafitte JJ, Houdret N, Pruvot FR, Lamblin G, Slayter HS, Roussel P. 1987. Peptides of human bronchial mucus glycoproteins. Size determination 
by electron microscopy and by biosynthetic experiments. Biochem J 248: 189-195.

Marszalek PE, Farrell B, Verdugo P, Fernandez JM. 1997. Kinetics of release of serotonin from isolated granules. (I) Amperometric detection of serotonin from electroporated granules. Biophys J 73: 1160-1168.

Matsui H, Grubb BR, Tarran R, Randell SH, Gatzy JT, Davis CW, Boucher RC. 1998. Evidence for periciliary liquid layer depletion, not abnormal ion composition, in the pathogenesis of cystic fibrosis airways disease. Cell 95: 1005-1015.

Matthews WG, Davis CW, Boucher RC. 2002. Atomic force microscopy studies of the structure of single mucin molecules and their viscoelastic properties. In Biophysical Society 46th Annual Meeting (Abstract 814 poster), San Francisco, CA. Biophysical Society, Bethesda, MD.

Meyer EE, Rosenberg KJ, Israelachvili J. 2006. Recent progress in understanding hydrophobic interactions. Proc Natl Acad Sci 43: 15739-15746.

Muchekehu RW, Quinton PM. 2010. A new role for bicarbonate secretion in cervico-uterine mucus release. J Physiol 588: 2329-2342.

Ohmine I, Tanaka T. 1982. Salt effects on the phase transitions of ionic gels. J Chem Phys 83: 89-99.

Perez-Villar J, Hill RL. 1999. The structure and assembly of secreted mucins. J Biol Chem 274: 31751-31754.

Potter JL, Mattheus LW, Spector W, Lemm J. 1967. Studies on pulmonary secretions. II: Osmolality, and ionic environment of pulmonary secretions from patients with cystic fibrosis, bronchiectasis, and laringectomy. Am Rev Resp Dis 96: 83-97.

Quinton PM. 2010. Role of epithelial $\mathrm{HCO}_{3}^{-}$transport in mucin secretion: Lessons from cystic fibrosis. Am J Physiol Cell Physiol 299: C1222-C1233.

Rayner C, Wilson R. 1997. Mucus-bacteria interactions. In Airway mucus: Basic mechanisms and clinical perspectives (ed. Rogers DF, et al.), pp. 210-226. Birkhauser Verlag, Basel, Switzerland.

Riordan JR. 2005. Assembly of functional CFTR chloride channels. Annu Rev Physiol 67: 701-718.

Round AN, Berry M, McMaster TJ, Stoll S, Gowers D, Corfield AP, Milles MJ. 2002. Heterogeneity and persistence length in human ocular mucins. Biophys J 83: 1661-1670.

Round AN, Berry M, McMaster TJ, Corfield AP, Milesa MJ. 2004. Glycopolymer charge density determines conformation in human ocular mucin gene products: An atomic force microscope study. J Struct Biol 145: 246-253.

Round AN, McMaster TJ, Miles MJ, Corfield AP, Berry M. 2007. The isolated MUC5AC gene product from human ocular mucin displays intramolecular conformational heterogeneity. Glycobiology 17: 578-585.

Sheehan JK, Thornton DJ. 2000. Heterogeneity and size distribution of gel-forming mucins. Methods Mol Biol 125: 87-96.

Sheehan JK, Oates K, Carlstedt I. 1984. Electron microscopy of cervical, gastric, and bronchial mucus glycoproteins. Biochem J 239: 147-153.

Sheehan JK, Howard M, Richardson PS, Longwill T, Thornton DJ. 1999. Physical characterization of a low-charge glycoform of the MUC5B mucin comprising the gel- phase of an asthmatic respiratory mucous plug. Biochem J 338: 507-513.

Sheppard DN, Welsh MJ. 2006. Structure and function of the CFTR chloride channel. Physiol Rev 79: 23-45.

Tam PY, Verdugo P. 1981. Control of mucus hydration as a Donnan equilibrium process. Nature 292: 340-342.

Tanaka T. 1981. Gels. Sci Am 244: 124-138.

Tanaka T, Fillmore DJ. 1979. Kinetics of swelling of gels. J Chem Phys 70: 1214-1218.

Tanaka T, Fillmore D, Sun S, Nishio I, Wislow GS, Shah A. 1980. Phase transitions in ionic gels. Phys Rev Lett 45: 1636.

Thornton DJ, Sheehan JK, Carlstedt I. 1991. Heterogeneity of mucus glycoproteins from cystic fibrotic sputum: Are there different families of mucins? Biochem J 276: $677-$ 682 .

Thornton DJ, Rousseau K, McGuckin A. 2008. Structure and function of the polymeric mucins in airways mucus. Annu Rev Physiol 70: 459-486.

van Loosdrecht MC, Lyklema J, Norde W, Zehnder AJ. 1990. Influence of interfaces on microbial activity. Microb Rev 54: 75-87.

Verdugo P. 1980. $\mathrm{Ca}^{2+}$-dependent hormonal stimulation of ciliary activity. Nature 283: 764-765.

Verdugo P. 1984. Hydration kinetics of exocytosed mucins in cultured secretory cells of the rabbit trachea: A new model. In Mucus and mucosa (Ciba Foundation Symposium) (ed. Nuget J, O’Connor M), Vol. 109, pp. 212-234. Pitman, London.

Verdugo P. 1990. Goblet cells and mucus secretion. Annu Rev Physiol 52: 157-176.

Verdugo P. 1991. Mucin exocytosis. Am Rev Resp Dis 144: S33-S37.

Verdugo P. 1994. Polymer gel phase transition in condensation-decondensation of secretory products. Adv Poly Sci 110: $145-156$.

Verdugo P. 1998. Polymer biophysics of mucus in cystic fibrosis. In Cilia mucus and mucociliary interactions (ed. Baum GL, et al.), pp. 167-190. Marcel Dekker, New York.

Verdugo P. Mucus supramolecular topology: An elusive riddle. Proc Natl Acad Sci (submitted).

Verdugo P, Tam PY, Butler J. 1983. Conformational structure of respiratory mucus studied by laser correlation spectroscopy. Biorheology 20: 223-230.

Verdugo P, Deyrup-Olsen L, Aitken ML, Villalon MJ, Johnson D. 1987a. Molecular mechanism of mucin secretion: The role of intragranular charge shielding. J Dent Res 66: 506-508.

Verdugo P, Aitken ML, Langley L, Villalon MJ. 1987b. Molecular mechanism of product storage and release in mucin secretion. II. The role of extracellular $\mathrm{Ca}^{2+}$. Biorheology 24: 625-633.

Verdugo P, Deyrup-Olsen I, Martin AW, Luchtel DL. 1992. Polymer gel phase transition: The molecular mechanism of product release in mucin secretion. In Swelling of polymer networks, NATO ASI Series H (ed. Karalis E), Vol. 64, pp. 671-681. Springer-Verlag, Heidelberg, Germany. 
P. Verdugo

Villalon M, Basbaum CB, Johnson DE, Verdugo P. 1988. Xray microanalysis of secretory granules from respiratory goblet cells. FASEB J 2: A958.

Viney C, Huber AE, Verdugo P. 1993a. Liquid crystalline order in mucus. Macromolecules 26: 852-257.

Viney C, Huber AE, Verdugo P. 1993b. Processing biological polymers in the liquid crystalline state. In Biodegradable polymers and packaging (ed. Ching C, et al.), pp. 209224. Technomics, Lancaster, PA.
Waigh TA, Papagiannopoulos A. 2010. Biological and biomimetic comb polyelectrolytes. Polymers 2: 57-70.

Widdicombe JG. 1987. Role of lipids in airway function. Eur J Respir Dis 153: 197-204.

Yakubov GE, Papagiannopoulos A, Rat E, Easton RL, Waigh TA. 2007. Molecular structure and rheological properties of short-side-chain heavily glycosylated porcine stomach mucin. Biomacromolecules 8: $3467-$ 3477. 


\section{$\&_{\mathrm{CSH}}^{\infty} \&$ Cold Spring Harbor

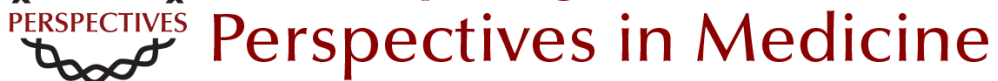

\section{Supramolecular Dynamics of Mucus}

Pedro Verdugo

Cold Spring Harb Perspect Med 2012; doi: 10.1101/cshperspect.a009597

Subject Collection Cystic Fibrosis

Antibiotic and Anti-Inflammatory Therapies for

Cystic Fibrosis

James F. Chmiel, Michael W. Konstan and J. Stuart Elborn

Structure and Function of the Mucus Clearance System of the Lung

Brenda M. Button and Brian Button

New Pulmonary Therapies Directed at Targets

Other than CFTR

Scott H. Donaldson and Luis Galietta

The Cystic Fibrosis Airway Microbiome

Susan V. Lynch and Kenneth D. Bruce

\section{Cystic Fibrosis Transmembrane Conductance \\ Regulator (ABCC7) Structure \\ John F. Hunt, Chi Wang and Robert C. Ford}

Status of Fluid and Electrolyte Absorption in

Cystic Fibrosis

M.M. Reddy and M. Jackson Stutts

The Influence of Genetics on Cystic Fibrosis

Phenotypes

Michael R. Knowles and Mitchell Drumm

Perspectives on Mucus Properties and Formation

--Lessons from the Biochemical World

Daniel Ambort, Malin E.V. Johansson, Jenny K.

Gustafsson, et al.
The Cystic Fibrosis Intestine

Robert C. De Lisle and Drucy Borowitz

Cystic Fibrosis Transmembrane Regulator

Correctors and Potentiators

Steven M. Rowe and Alan S. Verkman

The Cystic Fibrosis of Exocrine Pancreas

Michael Wilschanski and Ivana Novak

Dynamics Intrinsic to Cystic Fibrosis Transmembrane Conductance Regulator Function and Stability

P. Andrew Chong, Pradeep Kota, Nikolay V.

Dokholyan, et al.

The Cystic Fibrosis Gene: A Molecular Genetic

Perspective

Lap-Chee Tsui and Ruslan Dorfman

The CFTR Ion Channel: Gating, Regulation, and

Anion Permeation

Tzyh-Chang Hwang and Kevin L. Kirk

Assessing the Disease-Liability of Mutations in CFTR

Claude Ferec and Garry R. Cutting

Supramolecular Dynamics of Mucus Pedro Verdugo

For additional articles in this collection, see http://perspectivesinmedicine.cshlp.org/cgi/collection/ 